\title{
A study of the work stress and health of GDPs
}

\author{
'It's difficult being a dentist': stress and health in the general dental practitioner
}

H. L. Myers and L. B. Myers Br Dent J 2004; 197: 89-93

Objective The aim of the study was to investigate overall stress, work-stress and health in general dental practitioners (GDPs).

Design, setting and subjects A nationwide anonymous crosssectional survey was undertaken using stratified random sampling of 2,441 GDPs in the UK.

Outcome measures Measures included perceived stress, Work Stress Inventory for Dentists, job dissatisfaction, measures of health symptoms and health behaviour, dental and demographic information.

Results The main findings were that perceived stress was significantly correlated with measures of dental stress. Workrelated factors: fragility of dentist-patient relationship, time and scheduling pressures, staff and technical problems, job dissatisfaction, percentage NHS, and number of hours worked per week together explained nearly a half of GDPs overall stress in their life (linear multiple regression, adjusted $r^{2}=0.48, \mathrm{~F}(2,2404)=$ 509.68, $P<0.0001)$. Health behaviours such as alcohol use was associated with work stress $(r=0.18, P<0.001)$ and over a third of GDPs were overweight or obese. Sixty per cent of GDPs reported being nervy, tense or depressed, 58.3\% reported headache, 60\% reported difficulty in sleeping and 48.2\% reported feeling tired for no apparent reason. These were all related to work stress (one way analysis of variance, $\mathrm{F}(1,2211)=241.53 P<0.0001, \mathrm{~F}(1,2214)=$ $86.17 P<0.0001 ; \mathrm{F}(1,2215)=125.55 P<0.0001 ; \mathrm{F}(1,2211)=$ $209.67 P<0.0001$ respectively). Levels of minor psychiatric symptoms were high, with $32.0 \%$ of cases identified. The amount of backache was also high (reported by $68.3 \%$ of GDPs).

Conclusion A high percentage of NHS dentistry was associated with high levels of overall stress in GDPs' lives, indicating that the nature of NHS dentistry should be carefully investigated to try to improve GDPs working conditions. A comparatively large number of dentists reported high levels of psychological stress symptoms, such as being nervy, tense and depressed, showing minor psychiatric symptoms, with alcohol use being related to stress. Other factors reported which were not related to stress but may be related to the actual practice of dentistry were that a third of dentists were overweight or obese and over 60\% reported backache. Overall, these findings indicate the stressful nature of dentistry and difficulties in working conditions. The next step should be to develop interventions to help dentists to reduce stress in the dental surgery.

\section{IN BRIEF}

This paper found that:

- GDPs suffer from work-related stress.

- A lot of the GDP work-related stress is linked to working within the NHS.

- Minor psychiatric symptoms were high, similar to doctors.

- Over half of the GDPs reported backache, headache, difficulty in sleeping and being nervy, tense or depressed.

- A third of the GDPs were overweight or obese.

- This survey highlights the need to develop interventions to reduce GDPs' work-related stress and improve their working conditions.

\section{COMMENT}

The Health and Safety Executive recently ordered an NHS trust to carry out a risk assessment on staff stress. Staff had complained of bullying and excessive hours, and inspectors found management did not assess the risks from stress as part of their duty of care to their employees. This may have been the first enforcement notice to control workplace stress but it is unlikely to be the last. Stress has now become the most common reason for taking time off work overtaking back pain and influenza. Stress-related sickness is a particular problem in health, education and other public services.

Myers and Myers surveyed over 2,400 dentists and have provided us with a valuable up-to-date snapshot. It confirms the stressful nature of dental practice and analyses stressors and some of the contributory lifestyle issues. It adds further information to the 1996 study of dentists and dental staff carried out by Kay and Scarrott. ${ }^{1}$

The Health and Safety Executive define stress as 'the adverse reaction people have to excessive pressure' 2 and point out that stress is not a disease. However when that stress is intense and extended, it can lead to mental and physical ill health. The tabloids often present stress-related illness as a cloak for malingering. In reality, effective stress management can pay enormous dividends in improving the quality of our working lives. This applies just as much to the dental team as the dentist. Failure to take it seriously can have horrific consequences as the high rates of alcoholism, addiction and suicide in the profession demonstrate.

Their analysis highlights some of the problems in dentistry and will repay careful reading. They identify an occupation working under constant time pressures and financial constraints. They describe only too familiar scenarios of dealing with apprehensive patients, and the relentless pressure from a full waiting room when things are not going to plan. Not all stressors are surgery-based and balancing professional and home life is always going to be important. However they conclude that a half of overall stress is job related. They also note the classic stress symptoms such as feeling tense, tired, and having sleeping difficulties. Diet and exercise need greater attention because of the sedentary nature of the job.

It is clear that risk assessment in the future must include stress. Effective teamworking requires an overall assessment of pressure points and concerted action to address the problems. Those practices who have completed the 'Investors in People' programme have seen this happen. Simple time management can dramatically reduce stress in the whole practice. If you are always running late, you have a problem and reorganising your appointment book can work wonders. Finally this survey underlines the urgent need for action to reduce workplace stress.

A. Lawrence OBE,

Chair, Evidence-Based Dentistry Centre, doi:10.1038/sj.bdj.4811475

1. Kay E J, Scarrott D M. A survey of dental professionals' health and well-being. Br Dent J 1997; 183: 340-345.

2. Health and Safety Executive. A Short quide to work related stress. London: HSE Books. 